\title{
Metal Nanoclusters/Polyvinyl Alcohol Composite Films as the Alternatives for Fabricating Remote-Type White Light-Emitting Diodes
}

\author{
Zhaoyu Liu ${ }^{1}$, Dong Yao ${ }^{1, * \mathbb{D}}$, Huiwen Liu ${ }^{1,2}$ and Hao Zhang ${ }^{1,3, *}$ \\ 1 State Key Laboratory of Supramolecular Structure and Materials, College of Chemistry, Jilin University, \\ Changchun 130012, China; zhaoyul18@mails.jlu.edu.cn (Z.L.); liuhuiwenjlu@163.com (H.L.) \\ 2 Joint Laboratory of Opto-Functional Theranostics in Medicine and Chemistry, \\ The First Hospital of Jilin University, Changchun 130021, China \\ 3 Green Catalysis Center, College of Chemistry, Zhengzhou University, Zhengzhou 450001, China \\ * Correspondence: dongyao@jlu.edu.cn (D.Y.); hao_zhang@jlu.edu.cn (H.Z.)
}

check for updates

Citation: Liu, Z.; Yao, D.; Liu, H.; Zhang, H. Metal Nanoclusters/ Polyvinyl Alcohol Composite Films as the Alternatives for Fabricating Remote-Type White Light-Emitting Diodes. Nanomaterials 2022, 12, 204. https://doi.org/10.3390/ nano12020204

Academic Editors: Qiaofeng Yao, Xun Yuan, Zhennan Wu and Kosei Ueno

Received: 17 November 2021

Accepted: 6 January 2022

Published: 8 January 2022

Publisher's Note: MDPI stays neutral with regard to jurisdictional claims in published maps and institutional affiliations.

Copyright: () 2022 by the authors Licensee MDPI, Basel, Switzerland. This article is an open access article distributed under the terms and conditions of the Creative Commons Attribution (CC BY) license (https:// creativecommons.org/licenses/by/ $4.0 /)$.

\begin{abstract}
Packing luminescent metal nanoclusters (MNCs) into polymers and fabricating novel MNCs/polymer composite materials is effective in obtaining high-performance light-emitting diodes (LEDs). Herein, water soluble $\mathrm{Cu}$ and Au nanoclusters are encapsulated in polyvinyl alcohol (PVA) by a casting method. The obtained MNCs/PVA composite films are highly emissive with triple primary colors, and inherit the merits of PVA, such as transparency, flexibility, machinability, stability and self-healing ability. By employing the MNCs/PVA composite films as down-conversions, remote type monochromic and white LEDs are fabricated. The white LEDs (WLEDs) exhibit a maximum color rendering index (CRI) of 86 with a Commission Internationale de l'Eclairage (CIE) color coordinate of $(0.33,0.35)$. By varying the three MNCs/PVA film arrangement, the correlated color temperature (CCT) of the WLEDs is tuned from 5582 to $9490 \mathrm{~K}$, which signifies the possibility of MNCs/PVA as alternative light-emitting materials for advanced illumination and display in the future.
\end{abstract}

Keywords: metal nanoclusters; fluorescence; polyvinyl alcohol; composite film; light-emitting diodes

\section{Introduction}

Light-emitting nanomaterials, such as rare earth phosphors, colloidal quantum dots, and perovskites, embedder in resins, have been widely used as down-conversion layers in light-emitting diodes (LEDs) for illumination and display [1-6]. Despite the excellent performances in optical properties, these light-emitting materials still suffers from tedious production, high price and/or high toxicity [7-9]. To meet the requirements of "green development" concept, novel nontoxic, low cost and easily available light-emitting materials are greatly welcome [10-12].

Metal nanoclusters (MNCs), representatively, $\mathrm{Au}, \mathrm{Ag}$ and $\mathrm{Cu} \mathrm{NCs}$, are one of the ideal candidate lighting materials [13-17]. Composed of several to hundreds of metal atoms, these MNCs possess sizes close to the Fermi wavelength of electrons and exhibit molecular-like optical properties, such as discrete energy levels and strong and tunable photoluminescence (PL) [18-21]. Additionally, what is even more important is that these MNCs are heavy-metal-free and can be easily obtained. Even so, MNC-based LEDs still have two drawbacks: (i) MNCs possess less PL stability than traditional light-emitting materials, especially when exposed to the stimulus such as heat, oxygen and moisture [22]; (ii) the conventional LED structure is to coat the light-emitting materials embedded in resins onto the UV chips. The direct contact to the UV chips results in continuous heat, and further leads to the phase separation, color inhomogeneity and efficiency deterioration [23-27]. Packaging the MNCs into polymers offers the possibility to overcome the above-mentioned shortcomings [28-30]. 
Polyvinyl alcohol (PVA) is a commercialized polymer, which has been widely used as polarizer in the liquid crystal imaging system and approved by the USA Food and Drug Administration (FDA) for medical applications [31-34]. Apart from low cost and high biosafety, PVA also exhibits other advantages, such as compatibility, transparency, recyclability and ease of processing, which makes it a preferred host matrix for fabricating MNCs/polymer composite materials [35,36]. Packaging MNCs into MNCs/PVA composite materials, on the one hand, could protect MNCs from the external environmental stimulus, which improves the stability of the LED. On the other hand, the free-standing nature of the composite material make it possible to avoid the direct contact between MNCs and UV chips and build a remote-type structure, which could promote the light output performance of the LEDs [22].

In this work, 2-mercapto-1-methylimidazole capped $\mathrm{Cu}$ nanoclusters ( $\mathrm{Cu}-\mathrm{MMI} \mathrm{NCs}$ ), 6-Aza-2-thiothymidine capped Au nanoclusters (Au-ATT NCs) and bovine serum albumin capped Au nanoclusters (Au-BSA NCs) are cast into MNCs/PVA composite films with bright blue, green, and red emission, respectively. The MNCs/PVA composite films not only inherit the emissive property of the MNCs, but also possess the merits of high transparency, flexibility, stability and self-healing ability from PVA. As a proof of concept, the MNCs/PVA composite films are further employed as down-converters to build remotetype monochromic and white LEDs, which exhibit a maximum color rendering index (CRI) of 86 and correlated color temperature (CCT) ranging from 5582 to $9490 \mathrm{~K}$.

\section{Materials and Methods}

\subsection{Materials}

Copper chloride dihydrate $\left(\mathrm{CuCl}_{2} \cdot 2 \mathrm{H}_{2} \mathrm{O}, 99.99 \%\right)$, 2-mercapto-1-methylimidazole (MMI, 98.0\%), L-arginine (Arg, 99.0\%) and bovine serum albumin (BSA, 98.0\%) were purchased from Aladdin Reagent Company, Shanghai, China. 6-Aza-2-thiothymidine (ATT) was purchased from Alfa Aesar Chemical Co., Ltd., Shanghai, China. Chloroauric acid $\left(\mathrm{HAuCl}_{4} \cdot 4 \mathrm{H}_{2} \mathrm{O}, \mathrm{Au} \mathrm{mol} \%>47.8 \%\right)$ and sodium hydroxide $(\mathrm{NaOH})$ were purchased from Sinopharm Chemical Reagent Co., Ltd., Shanghai, China. Polyvinyl alcohol 1788 (PVA) was purchased from Chengdu Kelong Chemical Co., Ltd., Chengdu, China. All the materials were used as received without further purification.

\subsection{Preparation of $\mathrm{Cu}-\mathrm{MMI} N \mathrm{~N}$ s}

$\mathrm{Cu}-\mathrm{MMI}$ NCs were synthesized according to the previous report with slight modification. In summary, $14.0 \mathrm{mg}$ of $\mathrm{CuCl}_{2} \cdot 2 \mathrm{H}_{2} \mathrm{O}$ was dissolved in $5 \mathrm{~mL}$ of water and stirred until completely dissolved. Subsequently, $37.7 \mathrm{mg}$ of $\mathrm{MMI}$ and $118.8 \mathrm{mg}$ of $\mathrm{NaOH}$ were added under vigorous stirring. The reaction was maintained at $55^{\circ} \mathrm{C}$ in the water bath for $50 \mathrm{~min}$, and the obtained crude product was purified with centrifugation.

\subsection{Preparation of $A u-A T T$ NCs}

Au-ATT NCs were synthesized according to the previous report with slight modification. In summary, $5 \mathrm{~mL}$ of $\mathrm{HAuCl}_{4} \cdot 4 \mathrm{H}_{2} \mathrm{O}(10 \mathrm{mmol} / \mathrm{L})$ and $5 \mathrm{~mL}$ of ATT $(80 \mathrm{mmol} / \mathrm{L})$ were mixed and stirred for $2 \mathrm{~min}$. Subsequently, $0.05 \mathrm{~mol}$ of $\mathrm{NaOH}$ solution was added to adjust the $\mathrm{pH}$ to weakly alkaline. After stirring in the dark at room temperature for $1 \mathrm{~h}$, the obtained crude product was purified by dialysis. Then, $1 \mathrm{~mL}$ of Arg $(40 \mathrm{mmol} / \mathrm{L})$ was added to the as-prepared solution and the $\mathrm{pH}$ was adjusted to 10 . The solution was stirred at $37^{\circ} \mathrm{C}$ for $24 \mathrm{~h}$ until the Au-ATT NCs with bright green emission was obtained.

\subsection{Preparation of $A u-B S A N C s$}

To synthesize Au-BSA NCs, $5 \mathrm{~mL}$ of $\mathrm{HAuCl}_{4} \cdot 4 \mathrm{H}_{2} \mathrm{O}(10 \mathrm{mmol} / \mathrm{L})$ and $5 \mathrm{~mL}$ of BSA $(50 \mathrm{mg} / \mathrm{mL})$ were mixed and stirred for $2 \mathrm{~min}$. Subsequently, $0.5 \mathrm{~mL}$ of $\mathrm{NaOH}(1 \mathrm{~mol} / \mathrm{L})$ was added to adjust the $\mathrm{pH}$ to weakly alkaline. The reaction was maintained in a water bath at $37^{\circ} \mathrm{C}$ for $24 \mathrm{~h}$, and the obtained crude product was purified by dialysis. 


\subsection{Preparation of MNCs/PVA Composite Film}

Totals of $0.5 \mathrm{~mL}$ of the as-prepared MNC aqueous solution $(5 \sim 30 \mathrm{mg} / \mathrm{mL})$ and $1.5 \mathrm{~mL}$ of the PVA aqueous solution (mass fraction 5 12\%) were mixed at room temperature and stirred for $30 \mathrm{~min}$. Subsequently, the mixture was centrifuged at $2000 \mathrm{rpm}$ for $3 \mathrm{~min}$ to remove air bubbles. Then, the mixture was evenly dropped onto a cleaned glass slice and cured at room temperature.

\subsection{Characterization}

Transmission electron microscopy (TEM) was recorded on a JEM-2100F electron microscope (Hitachi, Tokyo, Japan). The dynamic light scattering was measured by a Zetasizer NanoZS (Malvern, Shanghai, China). UV-visible absorption spectra were recorded using a Shimadzu 3100 UV-vis spectrophotometer (Shimadzu, Tokyo, Japan). Fourier-transform infrared (FTIR) spectroscopy were performed with a VERTEX 80V FTIR instrument (Bruke, Karlsruhe, Germany). Photoluminescence spectroscopy was carried out on a Shimadzu RF-5301 PC spectrophotometer (Shimadzu, Tokyo, Japan). X-ray powder diffraction (XRD) investigation was performed on an Empyrean X-ray diffractometer (Malvern, Shanghai, China) using $\mathrm{Cu}$ K radiation $(\lambda=1.5418 \AA)$. Atomic force microscope (AFM) tapping mode measurements were performed on a Bruke scanning probe microscope (Bruke, Madison, WI, USA) using a rotated tapping mode etched silicon probe tip. SEM micrographs were measured by JEOL-FESEM 6700F electron microscope (JEOL, Tokyo, Japan) with a primary electron energy of $3 \mathrm{kV}$. Before imaging, the samples were sputter-coated with $2 \mathrm{~nm} \mathrm{Pt}$. The luminescence decay curves were measured on Edinburgh FLS920 (Techcomp, Shanghai, China) equipped with an integrating sphere (excited at $365 \mathrm{~nm}$ ). An FESEM 6700F electron microscope (JEOL, Tokyo, Japan) was used to observe the process of self-healing. The spectra of the LEDs were measured at room temperature in the open air with a Spectrascan PR-650 spectrophotometer (JADAK, New York, NY, USA) equipped with an integrating sphere. The CIE (Commission Internationale de L'Eclairage 1931) calorimeter system was used to identify the color of the light.

\section{Results and Discussion}

\subsection{Preparation and Optical Properties of MNCs/PVA Solutions}

To obtain the MNCs/PVA solutions, blue-emissive Cu-MMI NCs, green emissive $\mathrm{Au}-\mathrm{ATT}$ and red-emissive Au-BSA NCs were foremost synthesized according to previous reports [37-39]. All the MNCs are well dispersed with uniform size distribution (Figure S1). The average diameter of $\mathrm{Cu}-\mathrm{MMI}$, Au-ATT and Au-BSA NCs is 2.1, 2.6 and $2.4 \mathrm{~nm}$, respectively. Desired amount of MNCs were then blended with PVA solutions to form homogenous MNCs/PVA solutions, where the MNCs mass fraction is $2.5 \%$. Figure S2 illustrates the absorption and PL emission of the MNCs dispersed in aqueous solution and PVA solution. The photoluminescence emission quantum yields (PLQYs) of both the MNCs are measured to be $6.4,2.5$ and $5.3 \%$, respectively. The MNCs/PVA composite solutions retain the optical properties of the MNCs. Only the spectra of Au-ATT NCs/PVA solution and Au-BSA NCs/PVA solution show a red shift of a few nanometers compared with those of the Au-ATT NCs and Au-BSA NCs aqueous solution, which could be attributed to interactions between the MNCs and PVA molecules and the electronic environment variation of the MNCs (Table S1). As shown in Figure S3 and Table S2, the PL lifetime of the MNCs/PVA solutions is significantly improved compared with the MNCs aqueous solutions. On the incorporation of PVA, due to the interactions, the vibration, rotation and torsion motion of the MNCs are restricted, which reduces the possibility of non-radiation paths and enhances the radiation attenuation, thereby extending the PL lifetime.

\subsection{Structural and Optical Characterization of the MNCs/PVA Composite Films}

MNCs/PVA solutions were dehydrated into films by a casting method. The obtained composite films are freestanding and can be easily peeled off from the substrate. The scanning electron microscopy (SEM) equipped with energy-dispersive $\mathrm{X}$-ray spectroscopy 
(EDS) reveals that all the MNCs/PVA composite films are composed of three main elements, $\mathrm{Cu} / \mathrm{Au}, \mathrm{C}$ and $\mathrm{O}$ and the MNCs are homogenously dispersed in the PVA matrix (Figure 1). The average roughness of the Cu-MMI NCs/PVA, Au-BSA NCs/PVA and AuATT NCs/PVA films is 2.57, 1.86 and $1.11 \mathrm{~nm}$, respectively, implying the smooth surface of the composite films (Figure S4). The roughness of the Cu-MMI NCs/PVA film is a little higher than those of the Au-BSA NCs/PVA and Au-ATT NCs/PVA films, which can be ascribed to the higher degree of aggregation of $\mathrm{Cu}-\mathrm{MMI}$ NCs in PVA [40-42].
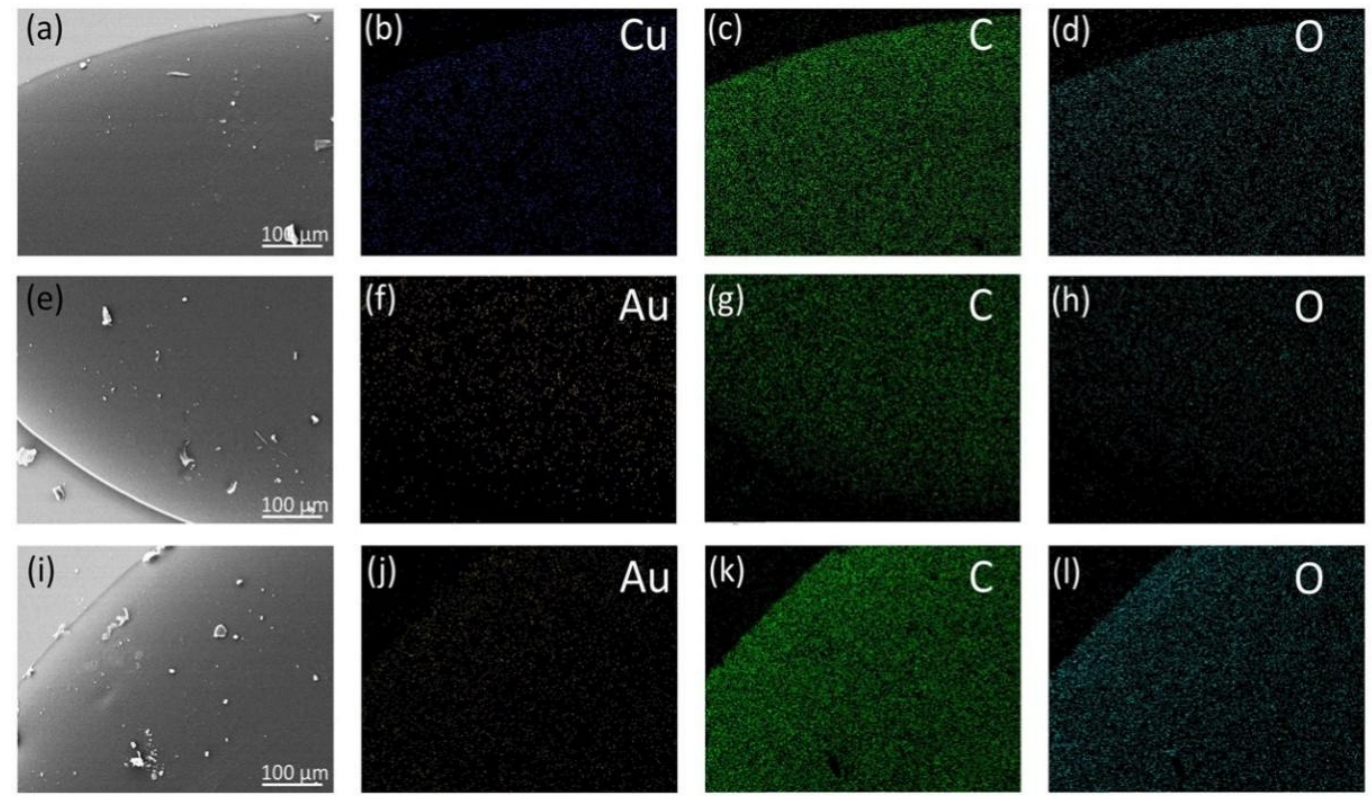

Figure 1. (a) SEM image and (b-d) SEM-EDS elemental mapping of Cu-MMI/PVA film, (e) SEM image and (f-h) SEM-EDS elemental mapping of Au-ATT/PVA film, and (i) SEM image and (j-1) SEM-EDS elemental mapping of Au-BSA/PVA film.

To explore the interaction between MNCs and PVA in the composite films, X-ray radiation diffraction (XRD) is performed. For the pure PVA film, there is a broad diffraction peak centered at 19.3 degrees, corresponding to the spacing of (101), which reflects the semi-crystalline characteristics of PVA (Figure S5). The semi-crystalline nature endows PVA with a mass of free voids between the polymeric chains in the amorphous phase, where the MNCs could become trapped. Compared with pure PVA, the XRD pattern of MNCs/PVA composites show no appearance of new crystalline peak, but slight red shifts, revealing that the MNCs are well-dispersed in PVA, and does not change the basic structural configuration of PVA. The distance decrement of the adjacent PVA molecules is ascribed to the interactions between the MNCs and PVA, probably with the hydroxyl groups $[43,44]$. The weak bonding effect in the MNCs/PVA composite is also affirmed by the FTIR analysis. The IR spectra of the MNCs/PVA composite films maintain the fundamental vibrational bands of PVA without the appearance of any additional peak (Figure S6). Comparatively, the characteristic stretching vibration peak of the hydroxyl group in PVA at $2947 \mathrm{~cm}^{-1}$ shifts to 2942 in Cu-MMI NCs/PVA, $2942 \mathrm{~cm}^{-1}$ in Au-ATT NCs/PVA and $2938 \mathrm{~cm}^{-1}$ in Au-BSA NCs/PVA, respectively [45]. These results indicate that no covalent bonds form between the MNCs and PVA and the incorporation of MNCs hardly affects the basic PVA structure, which is consistent with the XRD result.

The MNCs/PVA composite films are so transparent that printed letters beneath the MNCs/PVA films can be clearly seen (Figure $2 \mathrm{a}-\mathrm{c}$ ). With a $0.5 \%$ mass fraction of MNCs, the transmission of the three MNCs/PVA exceeds $70 \%$ in both the ultraviolet and visible range. When the MNC mass fraction is increased up to $2.5 \%$, Cu-MMI NCs/PVA films and AuATT NCs/PVA films achieve a transmission over $80 \%$ in the entire visible range, whereas the transmission of the Au-BSA NCs/PVA film remains over $70 \%$ in the range of 450 to 
$900 \mathrm{~nm}$ (Figure 2a-c). The minor absorption of visible light is beneficial for the fabrication of light-emitting diodes (LEDs). With the increment of the MNCs mass content, the PL intensity of the three MNCs/PVA composite films increase linearly, accompanied by the slight red shift of the PL peak position, which originates from the growing degree of MNCs aggregation (Figure 2d-f, Figures S1 and S7). The PLQYs of the MNCs/PVA films are 11.4, 10.4 and $9.9 \%$, and the average PL lifetimes are 10.7, 11.2 and $11.8 \mu \mathrm{s}$, respectively, which are significantly higher and longer than those of the corresponding MNCs (Figure S8). Based on the PLQYs and PL lifetimes, the radiative rate constant $\left(\mathrm{k}_{\mathrm{r}}\right)$ and nonradiative rate constant $\left(\mathrm{k}_{\mathrm{nr}}\right)$ of the MNCs and MNCs/PVA films are calculated (Table S3). After packing into the PVA films, the $\mathrm{k}_{\mathrm{r}}$ of MNCs is obviously promoted, whereas the $\mathrm{k}_{\mathrm{nr}}$ is inhibited. These phenomena are ascribed to the further vibration and rotation motion restriction of the MNCs in the PVA films.

(a)
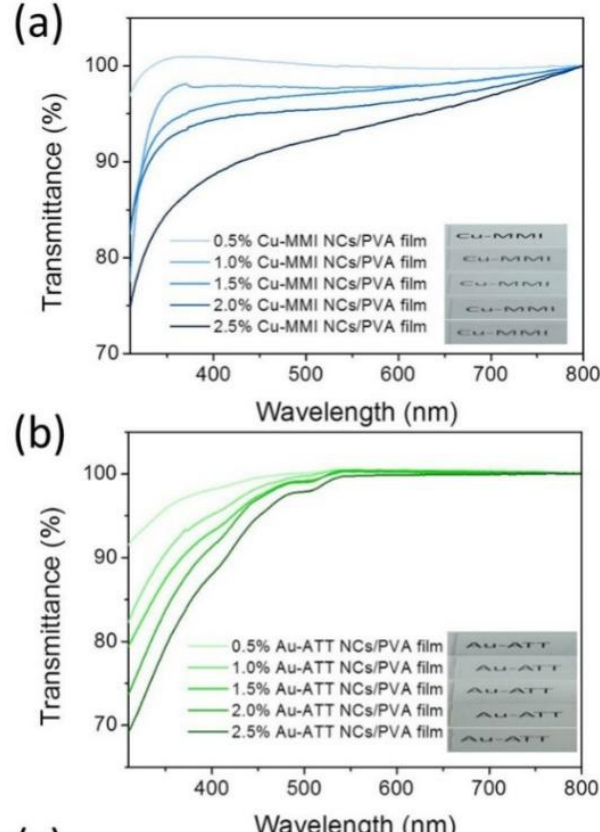

(c)

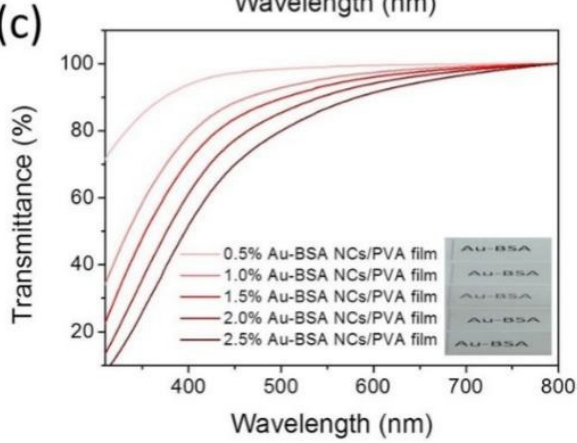

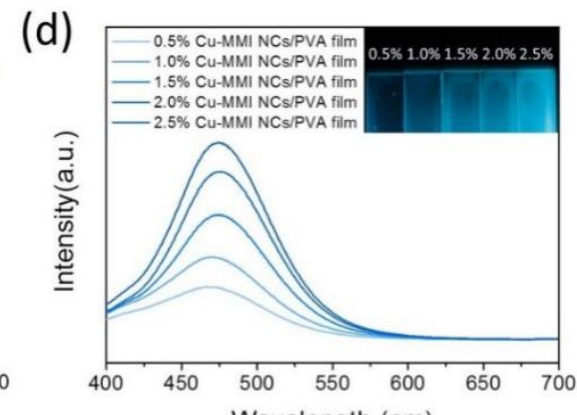

(e)

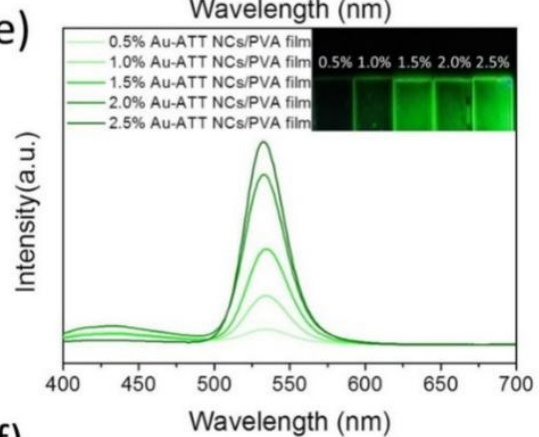

(f)

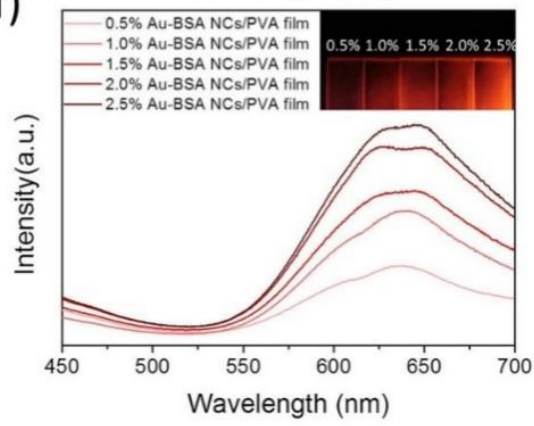

Figure 2. The transmission spectra of (a) Cu-MMI NCs/PVA film, (b) Au-ATT NCs/PVA film, and (c) Au-BSA NCs/PVA film with different NC concentrations. Insets of $(\mathbf{a}-\mathbf{c})$ exhibit the transparency of the composite films with corresponding photographs under room-light. The PL spectra of (d) Cu-MMI NCs/PVA film, (e) Au-ATT NCs/PVA film, and (f) Au-BSA NCs/PVA film with different NC concentrations. Insets are the corresponding photographs under excitation of $365 \mathrm{~nm}$.

\subsection{PL Stability of MNCs/PVA Composite Films}

PL stability is an important parameter for the practical application of photovoltaic and optoelectronic devices. Generally, MNCs are sensitive to environmental stimulus, which need to be stored in the dark at $4{ }^{\circ} \mathrm{C}$. Encapsulating MNCs into polymers can effectively improve the PL stability of MNCs to the environment. In Figure 3a, the MNCs/PVA composite films exhibit negligible PL intensity loss after stored in an open environment 
$\left(28^{\circ} \mathrm{C}\right.$, humidity $\left.=19 \%\right)$ for 14 days, and less than $20 \%$ PL intensity loss is recorded when the MNCs/PVA composite films are stored for 28 days, showing their good stability under ambient conditions. The MNCs/PVA composite films are heated to $80^{\circ} \mathrm{C}$ in air to explore the thermal stability (Figure 3b). After heating for $10 \mathrm{~h}$, more than $90 \%$ of PL intensity of the Au-ATT NCs/PVA and Au-BSA NCs/PVA composite films are preserved and the PL intensity of the $\mathrm{Cu}-\mathrm{MMI}$ NCs/PVA composite film is even improved beyond the initial intensity. As shown in Figure 3c, after irradiation under $365 \mathrm{~nm}$ for $10 \mathrm{~h}$, only minor changes in PL intensity of the MNCs/PVA composite films are recorded and more than $90 \%$ of PL intensity is preserved. Such good stability is attributed to the protection of PVA encapsulation to isolate the MNCs from the external environment, which is beneficial for the use of MNCs/PVA composite films in LEDs.
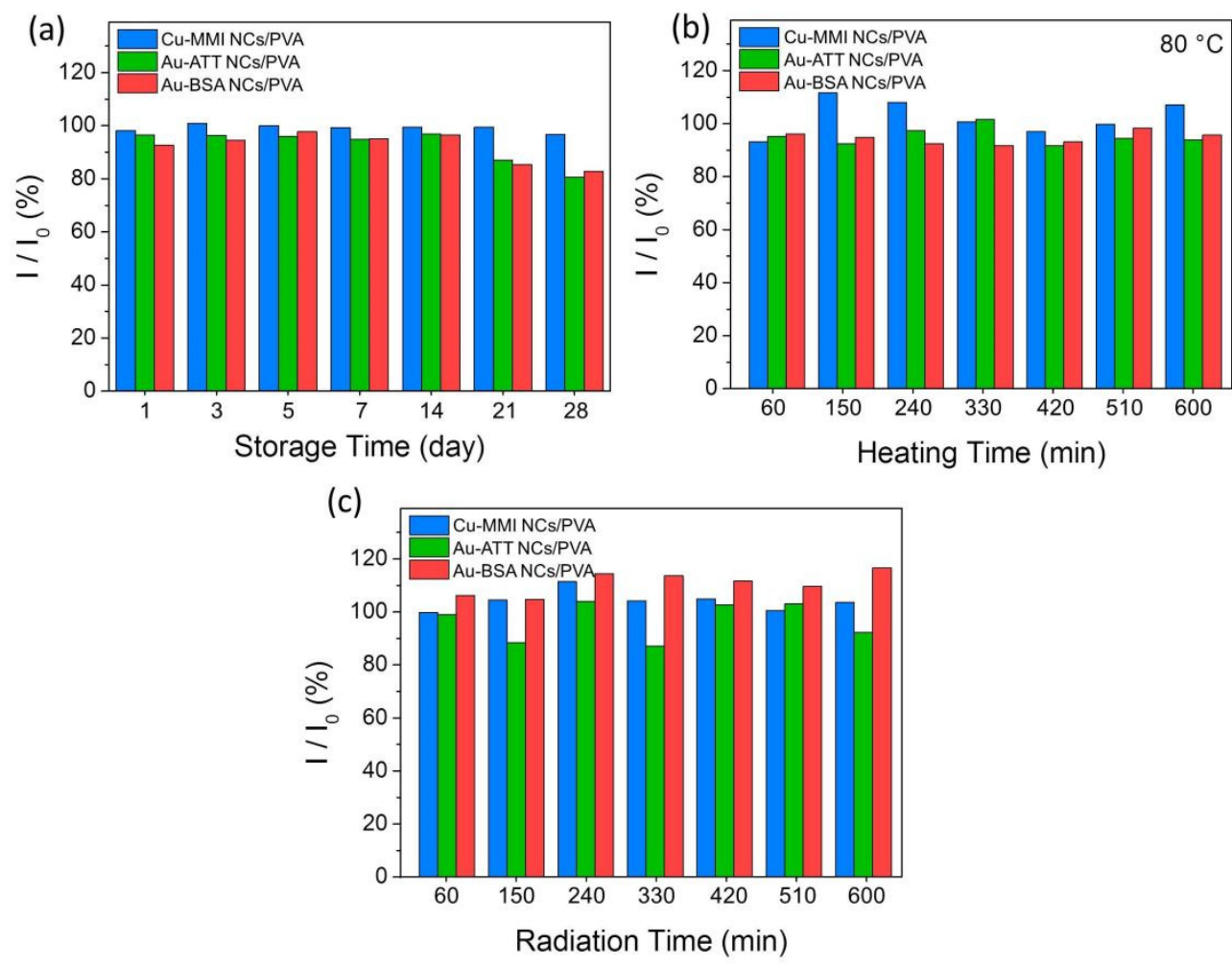

Figure 3. Stability of the Cu-MMI NCs/PVA film, Au-ATT NCs/PVA film, and Au-BSA NCs/PVA film: (a) storage stability, (b) thermal stability and (c) UV-irradiation stability.

\subsection{Fabricability and Self-Healing Ability of the MNCs/PVA Composite Films}

Due to the flexible and stretchable nature of PVA, it is easy for the MNCs/PVA composite films to be cast into any shapes with specific sizes. As shown in Figure 4a, a large square Au-BSA NCs/PVA film with a size of $16 \mathrm{~cm} \times 16 \mathrm{~cm}$, a coil of Au-ATT NCs/PVA composite film and a disk of Cu-MMI NCs/PVA composite film are fabricated. The three shaped MNCs/PVA composite films emit bright red, green and blue light, respectively, preserving their initial emission properties (Figure $4 \mathrm{~b}$ ).

Moreover, the MNCs/PVA films also inherit the self-healing properties of PVA. As illustrated in Figure $4 \mathrm{c}$, to repair the $10 \mu \mathrm{m}$ wide incision on the Cu-MMI NCs/PVA film, $1 \mu \mathrm{L}$ of water is dropped onto the incision. The incision narrows immediately and completely disappears in 5 min without external heating. The addition of the trace water promotes the intermolecular hydrogen bonding between adjacent PVA molecules and thus inducing the self-healing process. Based on the self-healing ability, two separated slices of $1 \mathrm{~cm} \times 1 \mathrm{~cm}$ Cu-MMI NCs/PVA film and Au-BSA NCs/PVA film are assembled through side-by-side arrangement and assistance of water, demonstrating the possibility of the MNCs/PVA films to build multicolor pixel arrays (Figure $4 \mathrm{~d}-\mathrm{g}$ ) [46]. 

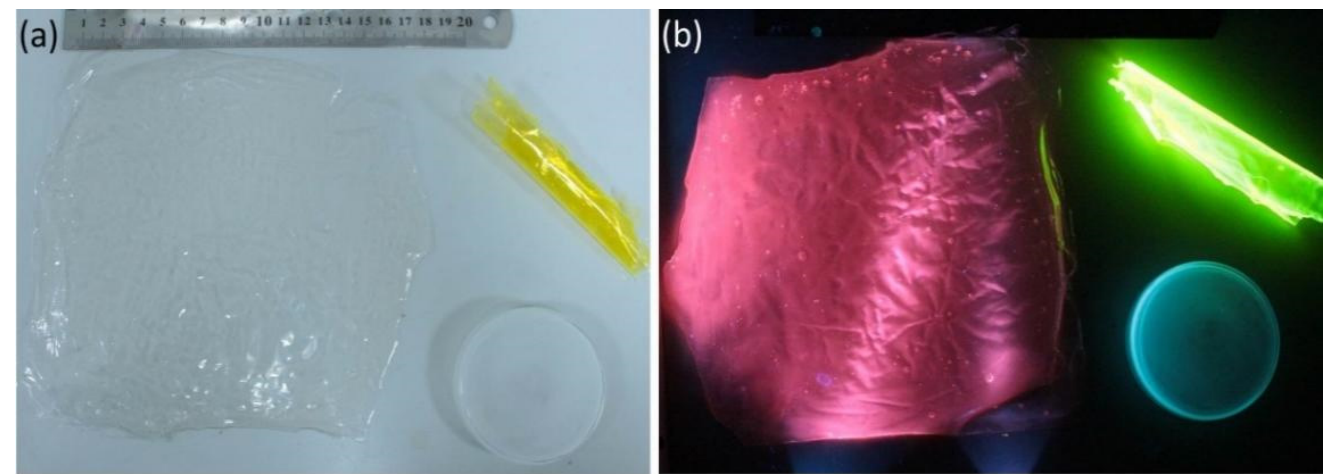

(c)
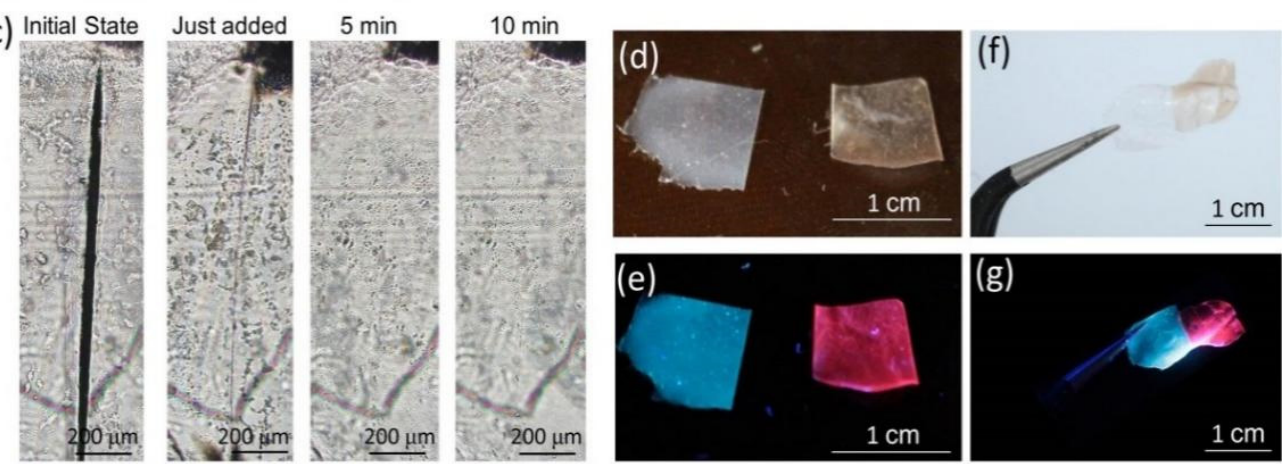

Figure 4. Photographs of the Cu-MMI NCs/PVA film, Au-ATT NCs/PVA film, and Au-BSA NCs/PVA film under (a) room light and (b) $365 \mathrm{~nm}$ excitation. (c) The light microscope photographs demonstrating the self-healing process of the Cu-MMI NCs/PVA film (from left to right). (d) The photographs of the separated Cu-MMI NCs/PVA and Au-BSA NCs/PVA films under room light and (e) under $365 \mathrm{~nm}$ excitation. (f) The photographs of the separated Cu-MMI NCs/PVA and Au-BSA NCs/PVA films under room light and (g) under $365 \mathrm{~nm}$ excitation.

\subsection{Application of MNCs/PVA Composite Films in Remote-Type LEDs}

Based on the properties discussed above, the MNCs/PVA composite films are considered to be excellent down-converter alternatives for LED application. Since the MNCs/PVA composite films are free standing, a remote-type LED architecture is applicable to avoid the direct contacts between the down-converters and the GaN chip, minimize the operating temperature and reduce phase segregation between MNCs and PVA, thus improving the LED performance (Figure S9). As shown in Figure 5a-c, a single layer of the MNCs/PVA film is employed to fabricate monochromic LEDs. The emission peaks of the blue, green and red LEDs are centered at $478 \mathrm{~nm}, 534 \mathrm{~nm}$ and $665 \mathrm{~nm}$, respectively (Figure $5 \mathrm{a}-\mathrm{c}$ ). The corresponding CIE color coordinates of the three LEDs are $(0.13,0.16),(0.31,0.67)$ and $(0.62$, 0.34 ), composing an RGB triangle which covers $80.1 \%$ of the NTSC color space (Figure $5 \mathrm{~h}$ ). To extend the emission color of the LED, a double layer of the MNCs is employed. As shown in Figure $5 \mathrm{~d}-\mathrm{f}$, pink, turquoise and orange LEDs are obtained with the combination of Cu-MMI NCs/PVA film and Au-BSA NCs/PVA film, Cu-MMI NCs/PVA film and AuATT NCs/PVA film, and Au-ATT NCs/PVA film and Au-BSA NCs/PVA film, respectively, with corresponding chromaticity coordinates at $(0.27,0.29),(0.21,0.40)$ and $(0.44,0.50)$ (Figure 5h).

To fabricate the WLED, triple-layer films are utilized together as the down-converter. The three composite films are arranged with a R-G-B sequence from bottom up, where $\mathrm{R}$ represents red-emissive Au-BSA NCs/PVA film, G represents green-emissive Au-ATT NCs/PVA film and B represents blue-emissive Cu-MMI NCs/PVA film. Figure $5 \mathrm{~g}$ exhibits the photograph and spectrum of the WLED. The chromaticity coordinate of the WLED is $(0.33,0.35)$, which is close to the pure white light coordinate $(0.33,0.33)$. The color rendering index (CRI) and correlated color temperature (CCT) of the WLED is 86 and $5582 \mathrm{~K}$, respectively, with a luminance of $89.36 \mathrm{~cd} / \mathrm{m}^{2}$. Note that the low efficiency is mainly 
due to the poor quality of the GaN chip. As is known to all, reabsorption exists between different emitters with absorption and emission overlaps. Based on the reabsorption effect, various composite film arrangements are attempted to tune the property of the WLED (Figure S10). Apart from the R-G-B sequence, other five combinations based on the three MNCs/PVA films are list in Table S4. The emission of the WLEDs is tuned from pure white to cold white, with CCT ranging from 5582 to $9490 \mathrm{~K}$ (Table S4).
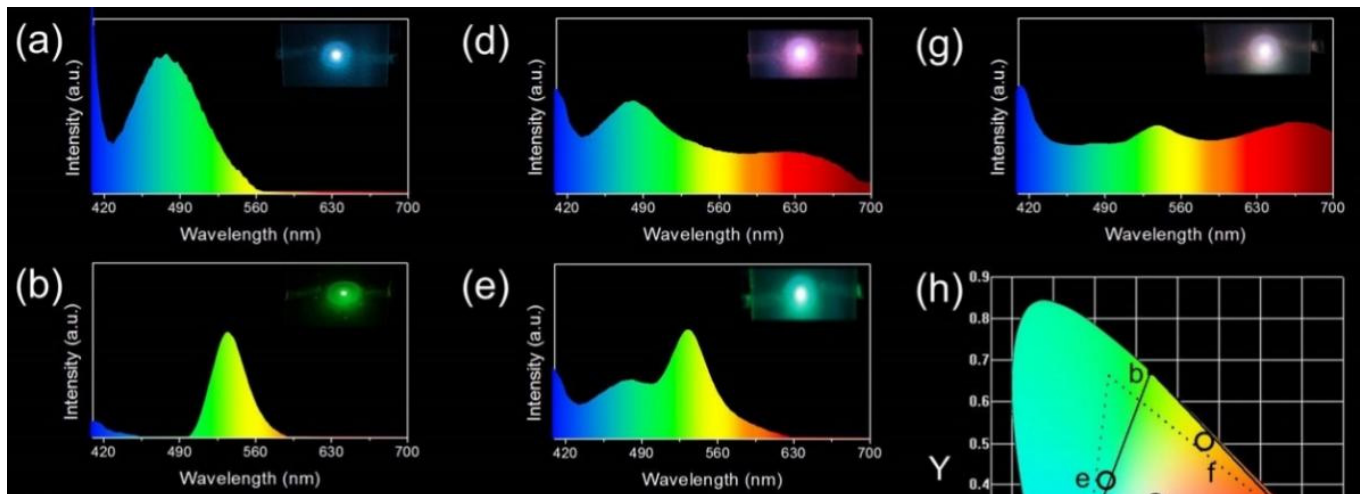

(e)
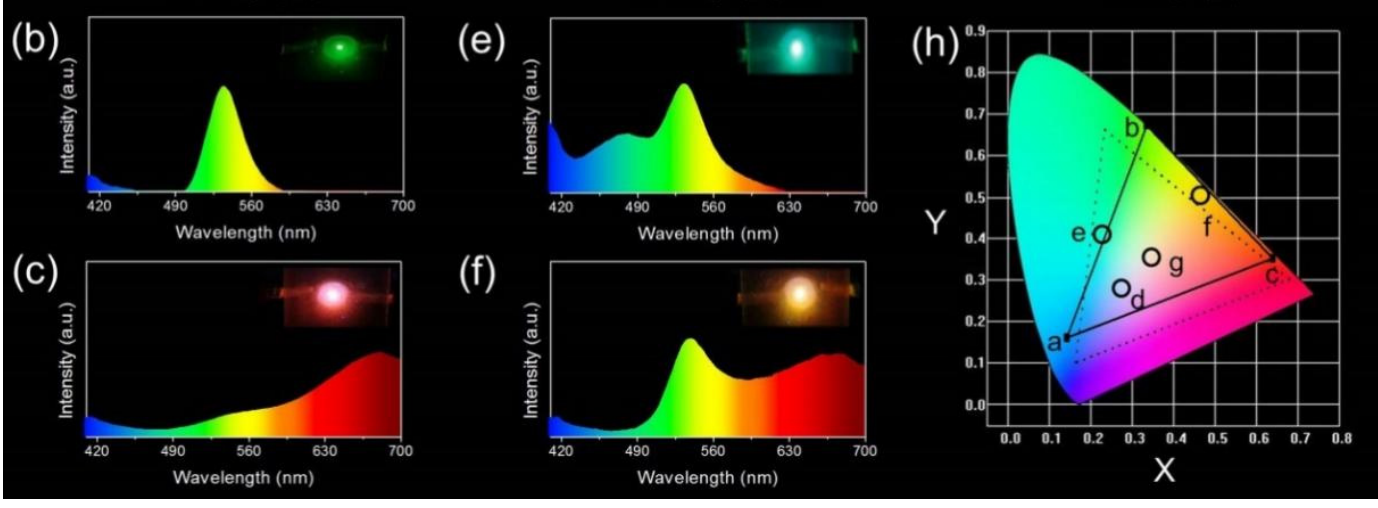

Figure 5. The emission spectra of the remote LEDs based on monolayer and multilayer MNCs/PVA films: (a) Cu-MMI/PVA film, (b) Au-ATT/PVA film, (c) Au-BSA/PVA film, (d) Cu-MMI/PVA film and Au-BSA/PVA film, (e) Cu-MMI/PVA film and Au-ATT/PVA film, (f) Au-ATT/PVA film and Au-BSA/PVA film and (g) Cu-MMI/PVA film, Au-ATT/PVA film, and Au-BSA/PVA film. Insets of $(\mathbf{a}-\mathbf{g})$ are the corresponding photographs of the remote LEDs. (h) The CIE chromaticity coordinates of the LEDs in $(\mathbf{a}-\mathbf{g})$. The dashed line is the range of color gamut of NTSC and the solid line is the color coverage of the LED based on our MNCs/PVA films.

\section{Conclusions}

In summary, we succeeded in fabricating free-standing $\mathrm{Cu}-\mathrm{MMI}$ NCs/PVA, AuATT NCs/PVA, Au-BSA NCs/PVA composite films by a simple casting method. The MNCs/PVA composite films are transparent and can emit three primary colors under $365 \mathrm{~nm}$ irradiation, respectively. Moreover, the MNCs/PVA composite films are easily processed into desired shapes and sizes, and exhibit excellent stability and self-healing ability. A remote-type WLED prototype with CIE coordinate of $(0.33,0.35)$ and high CRI of 86 is further fabricated by employing the MNCs/PVA composite films as down-converters, the CCT of which could be tuned through various MNCs/PVA film arrangements. Due to the nontoxicity and ease of processing, this work may promote the practical application of the luminescent MNCs/PVA composite films as down-converter alternatives for lighting.

Supplementary Materials: The following are available online at https: / www.mdpi.com/article / 10.3390/nano12020204/s1, Figure S1: High magnification TEM images of (a) Cu-MMI NCs, (b) Au-ATT NCs and (c) Au-BSA NCs; Figure S2: The absorption spectra of (a) Cu-MMI NC and Cu-MMI/PVA solution, (b) Au-ATT NC and Au-ATT/PVA solution, and (c) Au-BSA NC and Au-BSA/PVA solution. (d-f) The PL spectra of corresponding MNCs and MNCs/PVA solution; Table S1: The position of PL emission peaks of MNCs, MNCs/PVA composite solutions and MNCs/PVA composite films; Figure S3: PL decay curves of the MNCs, MNCs/PVA composite solutions and films, which are detected under $365 \mathrm{~nm}$ excitation. (a) Cu-MMI NCs, (b) Au-ATT NCs, and (c) Au-BSA NCs; Table S2: The PL lifetime of the MNCs and MNCs/PVA composite solutions detected at $365 \mathrm{~nm}$; Figure S4: 3D AFM images of the (a) Cu-MMI NCs/PVA film, (b) Au-ATT NCs/PVA film and (c) Au-BSA 
NCs/PVA film; Figure S5: XRD patterns of PVA film, Cu-MMI NCs/PVA film, Au-ATT NCs/PVA film, and Au-BSA NCs/PVA film; Figure S6: FTIR spectra of PVA, Cu-MMI NCs/PVA, Au-ATT NCs/PVA, and Au-BSA NCs/PVA composite films; Figure S7: The linear regression curves of the PL emission intensity of (a) Cu-MMI NCs/PVA, (b) Au-ATT NCs/PVA, and (c) Au-BSA NCs/PVA composite films versus the weight content of MNCs; Figure S8: PL decay curves of the MNCs/PVA composite films, which are detected under $365 \mathrm{~nm}$ excitation. (a) Cu-MMI NCs, (b) Au-ATT NCs, and (c) Au-BSA NCs. Table S3: Photophysical data of MNCs and MNCs/PVA composite films. Figure S9: The structure illustration of a remote-type LED; Figure S10: PL emission spectra of WLEDs based on the triple composite films with different arrangement of Cu-MMI NCs/PVA, Au-ATT NCs/PVA, and Au-BSA NCs/PVA films, where B represents blue, G represents green and R represents red. From bottom up: (a) B-G-R, (b) B-R-G, (c) G-R-B, (d) G-B-R and (e) R-B-G; Table S4: Index parameters of WLEDs based on the triple composite films with different arrangement of Cu-MMI NCs/PVA, $\mathrm{Au}-\mathrm{ATT}$ NCs/PVA, and Au-BSA NCs/PVA films from the bottom up.

Author Contributions: Conceptualization, H.Z.; methodology, H.Z. and D.Y.; investigation, Z.L. and H.L.; writing—original draft preparation, Z.L. and D.Y.; writing-review and editing, H.Z.; supervision, H.Z.; funding acquisition, H.Z. and D.Y. All authors have read and agreed to the published version of the manuscript.

Funding: This research was funded by the National key research and development program of China (2016YFB0401701), NSFC (21773088, 21902057), the China Postdoctoral Science Foundation (2021M691201, 2020TQ0115) and Special Project from MOST of China.

Conflicts of Interest: The authors declare no conflict of interest.

\section{References}

1. Dasog, M.; Kehrle, J.; Rieger, B.; Veinot, J.G. Silicon Nanocrystals and Silicon-Polymer Hybrids: Synthesis, Surface Engineering, and Applications. Angew. Chem. Int. Ed. Engl. 2016, 55, 2322-2339. [CrossRef] [PubMed]

2. Liu, H.; Liu, Z.; Xu, W.; Yang, L.; Liu, Y.; Yao, D.; Zhang, D.; Zhang, H.; Yang, B. Engineering the Photoluminescence of CsPbX 3 $(\mathrm{X}=\mathrm{Cl}, \mathrm{Br}$, and I) Perovskite Nanocrystals Across the Full Visible Spectra with the Interval of $1 \mathrm{~nm}$. ACS Appl. Mater. Interfaces 2019, 11, 14256-14265. [CrossRef] [PubMed]

3. Li, S.; Zha, T.; Gong, X.; Hu, Q.; Yu, M.; Wu, J.; Li, R.; Wang, J.; Chen, Y. Cu-Cd-Zn-S/ZnS Core/Shell Quantum Dot/Polyvinyl Alcohol Flexible Films for White Light-emitting Diodes. RSC Adv. 2020, 10, 24425-24433. [CrossRef]

4. Song, S.; Wang, X.; Wang, C.; Wen, Z.; Yang, Y.; Liu, H.; Yang, M.; Lin, Q. Large-Scale Synthesis of Flexible, Stable, and Transparent $\mathrm{MoS}_{2}$ Quantum Dots-Polyvinyl Alcohol Sensing Film. Part. Part. Syst. Charact. 2018, 35, 1800189. [CrossRef]

5. Xu, L.; Li, Y.; Gao, S.; Niu, Y.; Liu, H.; Mei, C.; Cai, J.; Xu, C. Preparation and Properties of Cyanobacteria-Based Carbon Quantum Dots/Polyvinyl Alcohol/ Nanocellulose Composite. Polymers 2020, 12, 1143. [CrossRef] [PubMed]

6. Yoon, H.C.; Kang, H.; Lee, S.; Oh, J.H.; Yang, H.; Do, Y.R. Study of Perovskite QD Down-Converted LEDs and Six-Color White LEDs for Future Displays with Excellent Color Performance. ACS Appl. Mater. Interfaces 2016, 8, 18189-18200. [CrossRef]

7. Wang, C.; Lin, H.; Xu, Z.; Huang, Y.; Humphrey, M.G.; Zhang, C. Tunable Carbon-Dot-Based Dual-Emission Fluorescent Nanohybrids for Ratiometric Optical Thermometry in Living Cells. ACS Appl. Mater. Interfaces 2016, 8, 6621-6628. [CrossRef]

8. Lei, J.; Huang, Z.; Gao, P.; Sun, J.; Zhou, L. Polyvinyl Alcohol Enhanced Fluorescent Sulfur Quantum Dots for Highly Sensitive Detection of $\mathrm{Fe}^{3+}$ and Temperature in Cells. Part. Part. Syst. Charact. 2021, 38, 2000332. [CrossRef]

9. Kalytchuk, S.; Polakova, K.; Wang, Y.; Froning, J.P.; Cepe, K.; Rogach, A.L.; Zboril, R. Carbon Dot Nanothermometry: Intracellular Photoluminescence Lifetime Thermal Sensing. ACS Nano 2017, 11, 1432-1442. [CrossRef]

10. Lai, W.F.; Wong, W.T.; Rogach, A.L. Development of Copper Nanoclusters for In Vitro and In Vivo Theranostic Applications. Adv. Mater. 2020, 32, 1906872. [CrossRef]

11. Jin, R.; Zeng, C.; Zhou, M.; Chen, Y. Atomically Precise Colloidal Metal Nanoclusters and Nanoparticles: Fundamentals and Opportunities. Chem. Rev. 2016, 116, 10346-10413. [CrossRef]

12. An, Y.; Ren, Y.; Bick, M.; Dudek, A.; Waworuntu, E.H.; Tang, J.; Chen, J.; Chang, B. Highly Fluorescent Copper Nanoclusters for Sensing and Bioimaging. Biosens. Bioelectron. 2020, 154, 112078. [CrossRef]

13. Wang, Z.; Chen, B.; Rogach, A.L. Synthesis, Optical Properties and Applications of Light-emitting Copper Nanoclusters. Nanoscale Horiz. 2017, 2, 135-146. [CrossRef]

14. Liu, X.; Astruc, D. Atomically Precise Copper Nanoclusters and Their Applications. Coord. Chem. Rev. 2018, 359, 112-126. [CrossRef]

15. Diez, I.; Ras, R.H. Fluorescent Silver Nanoclusters. Nanoscale 2011, 3, 1963-1970. [CrossRef] [PubMed]

16. Chen, L.Y.; Wang, C.W.; Yuan, Z.; Chang, H.T. Fluorescent Gold Nanoclusters: Recent Advances in Sensing and Imaging. Anal. Chem. 2015, 87, 216-229. [CrossRef] [PubMed]

17. Yuan, X.; Luo, Z.; Zhang, Q.; Zhang, X.; Zheng, Y.; Lee, J.Y.; Xie, J. Synthesis of Highly Fluorescent Metal (Ag, Au, Pt, and Cu) Nanoclusters by Electrostatically Induced Reversible Phase Transfer. ACS Nano 2011, 5, 8800-8808. [CrossRef] [PubMed] 
18. Shang, L.; Dong, S.; Nienhaus, G.U. Ultra-small Fluorescent Metal Nanoclusters: Synthesis and Biological Applications. Nano Today 2011, 6, 401-418. [CrossRef]

19. Rao, T.U.B.; Pradeep, T. Luminescent $\mathrm{Ag}_{7}$ and $\mathrm{Ag}_{8}$ Clusters by Interfacial Synthesis. Angew. Chem. Int. Ed. Engl. 2010, 49, 3925-3929.

20. Zhang, L.; Wang, E. Metal Nanoclusters: New Fluorescent Probes for Sensors and Bioimaging. Nano Today 2014, 9, 132-157. [CrossRef]

21. Zhao, Z.; Li, Y. Developing Fluorescent Copper Nanoclusters: Synthesis, Properties, and Applications. Colloids Surf. B 2020, 195, 111244. [CrossRef]

22. Wang, Z.; Xiong, Y.; Kershaw, S.V.; Chen, B.; Yang, X.; Goswami, N.; Lai, W.F.; Xie, J.; Rogach, A.L. In Situ Fabrication of Flexible, Thermally Stable, Large-Area, Strongly Luminescent Copper Nanocluster/Polymer Composite Films. Chem. Mater. 2017, 29, 10206-10211. [CrossRef]

23. Lin, H.; Wang, B.; Xu, J.; Zhang, R.; Chen, H.; Yu, Y.; Wang, Y. Phosphor-in-glass for High-powered Remote-type White AC-LED ACS Appl. Mater. Interfaces 2014, 6, 21264-21269. [CrossRef] [PubMed]

24. Peppas, N.A.; Hilt, J.Z.; Khademhosseini, A.; Langer, R. Hydrogels in Biology and Medicine: From Molecular Principles to Bionanotechnology. Adv. Mater. 2006, 18, 1345-1360. [CrossRef]

25. Song, Y.H.; Ji, E.K.; Bak, S.H.; Kim, Y.N.; Lee, D.B.; Jung, M.K.; Jeong, B.W.; Yoon, D.H. New Design of Hybrid Remote Phosphor with Single-layer Graphene for Application in High-power LEDs. Chem. Eng. J. 2016, 287, 511-515. [CrossRef]

26. Wang, Z.; Chen, B.; Zhu, M.; Kershaw, S.V.; Zhi, C.; Zhong, H.; Rogach, A.L. Stretchable and Thermally Stable Dual Emission Composite Films of On-Purpose Aggregated Copper Nanoclusters in Carboxylated Polyurethane for Remote White Light-Emitting Devices. ACS Appl. Mater. Interfaces 2016, 8, 33993-33998. [CrossRef]

27. Zhang, X.; Wang, J.; Huang, L.; Pan, F.; Chen, Y.; Lei, B.; Peng, M.; Wu, M. Tunable Luminescent Properties and ConcentrationDependent, Site-Preferable Distribution of $\mathrm{Eu}^{2+}$ Ions in Silicate Glass for White LEDs Applications. ACS Appl. Mater. Interfaces 2015, 7, 10044-10054. [CrossRef] [PubMed]

28. Hu, T.; Wen, Z.; Wang, C.; Thomas, T.; Wang, C.; Song, Q.; Yang, M. Temperature-controlled Spectral Tuning of Full-color Carbon Dots and Their Strongly Fluorescent Solid-state Polymer Composites for Light-emitting Diodes. Nanoscale Adv. 2019, 1, 1413-1420. [CrossRef]

29. Zeng, Q.; Zhang, X.; Feng, X.; Lu, S.; Chen, Z.; Yong, X.; Redfern, S.A.T.; Wei, H.; Wang, H.; Shen, H.; et al. Polymer-Passivated Inorganic Cesium Lead Mixed-Halide Perovskites for Stable and Efficient Solar Cells with High Open-Circuit Voltage over 1.3 V. Adv. Mater. 2018, 30, 1705393. [CrossRef] [PubMed]

30. Qu, F.; Zhang, S.; Huang, C.; Guo, X.; Zhu, Y.; Thomas, T.; Guo, H.; Attfield, J.P.; Yang, M. Surface Functionalized Sensors for Humidity-Independent Gas Detection. Angew. Chem. Int. Ed. 2021, 60, 6561-6566. [CrossRef] [PubMed]

31. Chong, S.F.; Smith, A.A.; Zelikin, A.N. Microstructured, functional PVA Hydrogels through Bioconjugation with Oligopeptides under Physiological Conditions. Small 2013, 9, 942-950. [CrossRef]

32. Hu, Z.; Ma, Z.; Peng, M.; He, X.; Zhang, H.; Li, Y.; Qiu, J. Composite Film Polarizer Based on the Oriented Assembly of Electrospun Nanofibers. Nanotechnology 2016, 27, 135301. [CrossRef]

33. Shin, E.J.; Lyoo, W.S.; Lee, Y.H. Polarizer Effect and Structure of Iodinated Before and After Casting Poly(vinyl alcohol) Film. J. Appl. Polym. Sci. 2011, 120, 397-405. [CrossRef]

34. Yao, R.; You, Q.; Liu, P.; Xu, Y. Synthesis and pH-induced Phase Transition Behavior of PAA/PVA Nanogels in Aqueous Media. J. Appl. Polym. Sci. 2009, 111, 358-362. [CrossRef]

35. Kamoun, E.A.; Kenawy, E.S.; Chen, X. A Review on Polymeric Hydrogel Membranes for Wound Dressing Applications: PVAbased Hydrogel Dressings. J. Adv. Res. 2017, 8, 217-233. [CrossRef] [PubMed]

36. Alipoori, S.; Mazinani, S.; Aboutalebi, S.H.; Sharif, F. Review of PVA-based Gel Polymer Electrolytes in Flexible Solid-state Supercapacitors: Opportunities and Challenges. J. Energy Storage 2020, 27, 101072. [CrossRef]

37. Deng, H.; Zhuang, Q.; Huang, K.; Balasubramanian, P.; Lin, Z.; Peng, H.; Xia, X.; Chen, W. Solid-state Thiolate-stabilized Copper Nanoclusters with Ultrahigh Photoluminescence Quantum Yield for White Light-emitting Devices. Nanoscale 2020, 12, 15791-15799. [CrossRef]

38. Deng, H.; Shi, X.; Wang, F.; Peng, H.; Liu, A.; Xia, X.; Chen, W. Fabrication of Water-Soluble, Green-Emitting Gold Nanoclusters with a 65\% Photoluminescence Quantum Yield via Host-Guest Recognition. Chem. Mater. 2017, 29, 1362-1369. [CrossRef]

39. Xie, J.; Zheng, Y.; Ying, J. Protein-Directed Synthesis of Highly Fluorescent Gold Nanoclusters. J. Am. Chem. Soc. 2009, 131, 888-889. [CrossRef]

40. Peng, Z.; Kong, L.X. Morphology of Self-assembled Polyvinyl Alcohol/Silica Nanocomposites Studied with Atomic Force Microscopy. Polym. Bull. 2007, 59, 207-216. [CrossRef]

41. Ingrosso, C.; Fakhfouri, V.; Striccoli, M.; Agostiano, A.; Voigt, A.; Gruetzner, G.; Curri, M.L.; Brugger, J. An Epoxy Photoresist Modified by Luminescent Nanocrystals for the Fabrication of 3D High-Aspect-Ratio Microstructures. Adv. Funct. Mater. 2007, 17, 2009-2017. [CrossRef]

42. Bhushan, B.; Qi, J. Phase Contrast Imaging of Nanocomposites and Molecularly Thick Lubricant Films in Magnetic Media. Nanotechnology 2003, 14, 886-895. [CrossRef] 
43. Sreeja, S.; Sreedhanya, S.; Smijesh, N.; Philip, R.; Muneera, C.I. Organic Dye Impregnated Poly(vinyl alcohol) Nanocomposite as An Efficient Optical Limiter: Structure, Morphology and Photophysical Properties. J. Mater. Chem. C 2013, 1, $3851-3861$. [CrossRef]

44. Bhajantri, R.F.; Ravindrachary, V.; Harisha, A.; Crasta, V.; Nayak, S.P.; Poojary, B. Microstructural Studies on BaCl 2 Doped Poly(vinyl alcohol). Polymer 2006, 47, 3591-3598. [CrossRef]

45. Shi, Y.; Zhuang, X.; Cao, L.; Gou, S.; Xiong, Y.; Lai, W.-F.; Wang, Z.; Rogach, A.L. Copper-Nanocluster-Based Transparent Ultraviolet-Shielding Polymer Films. ChemNanoMat 2019, 5, 110-115. [CrossRef]

46. Xiong, Y.; Zhu, M.; Wang, Z.; Schneider, J.; Huang, H.; Kershaw, S.V.; Zhi, C.; Rogach, A.L. A Building Brick Principle to Create Transparent Composite Films with Multicolor Emission and Self-Healing Function. Small 2018, 14, 1800315. [CrossRef] 